\title{
BMJ Open Leisure-time physical activity and circulating 25-hydroxyvitamin D levels in cancer survivors: a cross-sectional analysis using data from the US National Health and Nutrition Examination Survey
}

\author{
Lin Yang, ${ }^{1,2}$ Adetunji T Toriola ${ }^{1}$
}

To cite: Yang L, Toriola AT. Leisure-time physical activity and circulating 25-hydroxyvitamin D levels in cancer survivors: a cross-sectional analysis using data from the US National Health and Nutrition Examination Survey. BMJ Open 2017;7:e016064. doi:10.1136/ bmjopen-2017-016064

\section{- Prepublication history} and additional material for this paper are available online. To view these files please visit the journal online (http://dx.doi.org/ 10.1136/ bmjopen-2017-016064).

Received 25 January 2017 Revised 24 April 2017 Accepted 16 May 2017

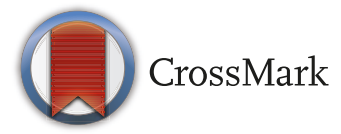

${ }^{1}$ The Division of Public Health Sciences, Department of Surgery, Washington University School of Medicine and Siteman Cancer Center, St Louis,

Missouri, USA

${ }^{2}$ Department of Epidemiology, Medical University of Vienna, Vienna, Austria

Correspondence to

Dr Adetunji T Toriola; toriolaa@ wudosis.wustl.edu

\section{ABSTRACT}

Objectives Circulating 25-hydroxyvitamin D (25-OHD) is associated with improved cancer prognosis in some studies, yet it may be a surrogate marker for physical activity. We investigated the associations of leisure-time physical activity (LTPA) with circulating 25-OHD levels in cancer survivors, and determined whether associations differ by indoor and outdoor activity.

Design Cross-sectional study.

Setting The US National Health and Nutrition Examination Survey (NHANES).

Participants Cancer survivors with available data on demographic information, measures of adiposity, smoking history, self-reported LTPA and circulating 25-OHD levels in five waves of NHANES (2001-2010).

Main outcomes measures Circulating 25-OHD levels. Results Multivariable linear regression and logistic regression models were used to evaluate the associations of self-reported LTPA with 25-OHD, adjusting for potential confounders. Due to the differences in LTPA measure, the analyses were conducted separately for 2001-2006 and 2007-2010 data. We further estimated associations by indoor and outdoor activity in the 2001-2006 data. There were 1530 cancer survivors (mean age $=60.5$ years, mean body mass index $\left.=28.6 \mathrm{~kg} / \mathrm{m}^{2}\right)$. The prevalent cancer sites were breast $(19.3 \%)$, prostate $(18.8 \%)$, cervix $(10.4 \%)$ and colon (8.6\%). Compared with inactive cancer survivors, being physically active was associated with higher circulating $25-\mathrm{OHD}$ levels $(8.07 \mathrm{nmol} / \mathrm{L}, 95 \% \mathrm{Cl}$ 4.63 to 11.52) for 2001-2006 data. In the mutually adjusted model, higher outdoor activity $(5.83 \mathrm{nmol} / \mathrm{L}$, $95 \% \mathrm{Cl} 1.64$ to 10.01$)$, but not indoor activity $(2.93 \mathrm{nmol} / \mathrm{L}$, $95 \% \mathrm{Cl}-1.80$ to 7.66$)$, was associated with statistically significantly higher 25-OHD levels. The interaction between indoor and outdoor activities was, however, not significant $(p=0.29)$. The only statistically significant association seen in the 2007-2010 data was among obese cancer survivors.

Conclusion Physical activity, particularly outdoor activity, is associated with higher 25-OHD levels in cancer survivors. In view of the possible beneficial effects of vitamin $D$ on cancer prognosis, engaging in outdoor
Strengths and limitations of this study

- To the best of our knowledge, this is the first study to investigate the association of leisure-time physical activity (LTPA) with circulating 25-hydroxyvitamin D (25-OHD) levels in cancer survivors. We further compared associations by outdoor and indoor LTPA.

- The current study pooled data from cancer survivors in a nationally representative adult sample in the USA.

- This study controlled for a range of factors that are known to affect circulating 25-OHD levels.

- Study limitations include the following: (1) the crosssectional nature makes it impossible to determine a causal association; (2) season, an important determinant of $25-\mathrm{OHD}$ levels, was categorised into two (winter and summer, rather than winter, summer, fall and spring); and (3) physical activity was self-reported.

physical activity could provide clinically meaningful increases in 25-OHD levels among cancer survivors.

\section{BACKGROUND}

There are $>15.5$ million cancer survivors in the USA and the number is expected to rise to 20 million by 2026 . $^{1}$ Identifying factors, particularly modifiable factors, that improve prognosis and survival in this rapidly expanding demographic group is, therefore, a high priority.

There is emerging evidence that vitamin D status is associated with improved cancer prognosis and survival, particularly colorectal and breast cancers. ${ }^{2-5}$ Circulating 25-hydroxyvitamin D (25-OHD) is the best indicator of overall vitamin $\mathrm{D}$ status because it has a long half-life, is unregulated by homeostatic systems in the body and reflects total vitamin 
D from multiple determinants. ${ }^{6-9}$ However, it has been suggested that circulating 25-OHD level may be a surrogate or biological marker for lifestyle factors that impact cancer prognosis, notably physical activity. ${ }^{2} 1011$ Physical activity, before and after cancer diagnosis, is associated with reduced mortality in cancer survivors, ${ }^{12-14}$ although the underlying mechanisms are still being elucidated. In cancer-free population, leisure-time physical activity (LTPA) is associated with an increase in circulating 25-OHD levels, which is thought to reflect exposure to sunlight, a major determinant of circulating 25-OHD levels. ${ }^{15}$ In support, studies have reported higher 25-OHD levels for the same amount of outdoor compared with indoor physical activity, ${ }^{16}$ although others have not. ${ }^{17}$

Nevertheless, it has also been shown that physical activity and sun exposure may have independent effects on circulating 25-OHD levels, suggesting that indoor physical activity might be sufficient to increase circulating 25-OHD levels through its effect on 25-OHD metabolism, such as 1,25-dihydroxyvitamin. ${ }^{18-21}$

To the best of our knowledge, no study has investigated the associations of physical activity with circulating 25-OHD levels in cancer survivors. Because physical activity declines after cancer diagnosis, findings in cancerfree population may not apply to cancer survivors. Using data from the National Health and Nutrition Examination Survey (NHANES), our objectives are to (1) investigate the associations of LTPA with circulating 25-OHD levels in cancer survivors, and (2) determine whether associations differ by indoor and outdoor physical activity. Study findings could have implications for public health recommendations in cancer survivors because physical inactivity and vitamin $\mathrm{D}$ insufficiency are prevalent among cancer survivors. $^{22} 23$

\section{METHODS}

\section{Study population}

The NHANES was designed to provide cross-sectional estimates on the prevalence of health, nutrition and potential risk factors among the civilian non-institutionalised US population up to 85 years of age. ${ }^{24}$ In brief, NHANES surveys a nationally representative complex, stratified, multistage, probability clustered sample of about 5000 participants each year in 15 counties across the country. The NHANES obtained approval from the National Center for Health Statistics Research Ethics Review Board and the participants provided written consent.

We extracted demographic information, measures of adiposity, smoking history, self-reported LTPA, circulating 25-OHD levels and cancer diagnosis, and combined them into a single data set for each data collection wave. Participants were considered as cancer survivors if they answered 'yes' to the question 'Have you ever been told by a doctor or other health professional that you had cancer or a malignancy of any kind?' We excluded participants who had non-melanoma skin cancer. This interview question was only given to men and women 20 years or older, and subsequently restricted the analysed sample to adult cancer survivors. We created a single data set for each wave of data from NHANES in 2001-2002, 2003-2004, 2005-2006, 2007-2008 and 2009-2010, and excluded those who were never diagnosed with cancer or were pregnant (figures 1 and 2).

\section{Circulating 25-OHD levels}

The process of blood collection is detailed in the NHANES Laboratory/Medical Technologist Procedures Manual. ${ }^{25}$ Participants who received chemotherapy within the last 4 weeks were excluded from blood collection in the NHANES study. Blood samples were collected, processed, stored and shipped to University of Washington, Seattle for testing. The lab method measuring 25-OHD for 20072010 changed from 2005 to 2006 and earlier in NHANES, and has been described previously. ${ }^{26}$ Briefly, circulating 25-OHD concentrations were measured at the National Center for Environmental Health, Centers for Disease Control and Prevention, Atlanta, Georgia, using the DiaSorin RIA kit (Stillwater, Minnesota, USA) between 2001 and 2006. We converted the 25-OHD data in 20012006 using provided regression to equivalent 25-OHD measurement from a standardised liquid chromatography-tandem mass spectrometry method, which was used in the analysis of 25-OHD in NHANES 2007-2010 data. This standardisation procedure therefore ensures that 25-OHD data are comparable between 2001-2006 and 2007-2010.

\section{Sociodemographic characteristics}

Sociodemographic characteristics including age, sex, race and ethnicity, and smoking status were extracted. Based on self-reported race and ethnicity, participants were classified into one of the three racial/ethnic groups: non-Hispanic white, non-Hispanic black, and Hispanic and others. We classified participants into three groups: never smokers (did not smoke 100 cigarettes and do not smoke now), former smokers (smoked 100 cigarettes in life and do not smoke now) and current smokers (smoked 100 cigarettes in life and smoke now).

\section{Body mass index}

Weight and height were measured at the time of physical examination in a mobile examination centre (MEC) or in the participant's home. The measurements followed standard procedures and were carried out by trained technicians using standardised equipment. Body mass index (BMI) was calculated as weight in $\mathrm{kg}$ / (height in metres). ${ }^{2}$ We categorised study participants into standard BMI categories: underweight $\left(<18.5 \mathrm{~kg} / \mathrm{m}^{2}\right)$, normal weight $\left(18.5-24.9 \mathrm{~kg} / \mathrm{m}^{2}\right)$, overweight $(25.0-29.9 \mathrm{~kg} /$ $\left.\mathrm{m}^{2}\right)$ and obese $\left(\geq 30.0 \mathrm{~kg} / \mathrm{m}^{2}\right)$. For analytic purposes, we combined those who were underweight and those who had normal weight into one category $\left(\leq 25 \mathrm{~kg} / \mathrm{m}^{2}\right)$.

\section{Season of blood draw}

Blood samples were collected at the time of physical examination in an MEC or in the participant's home. 


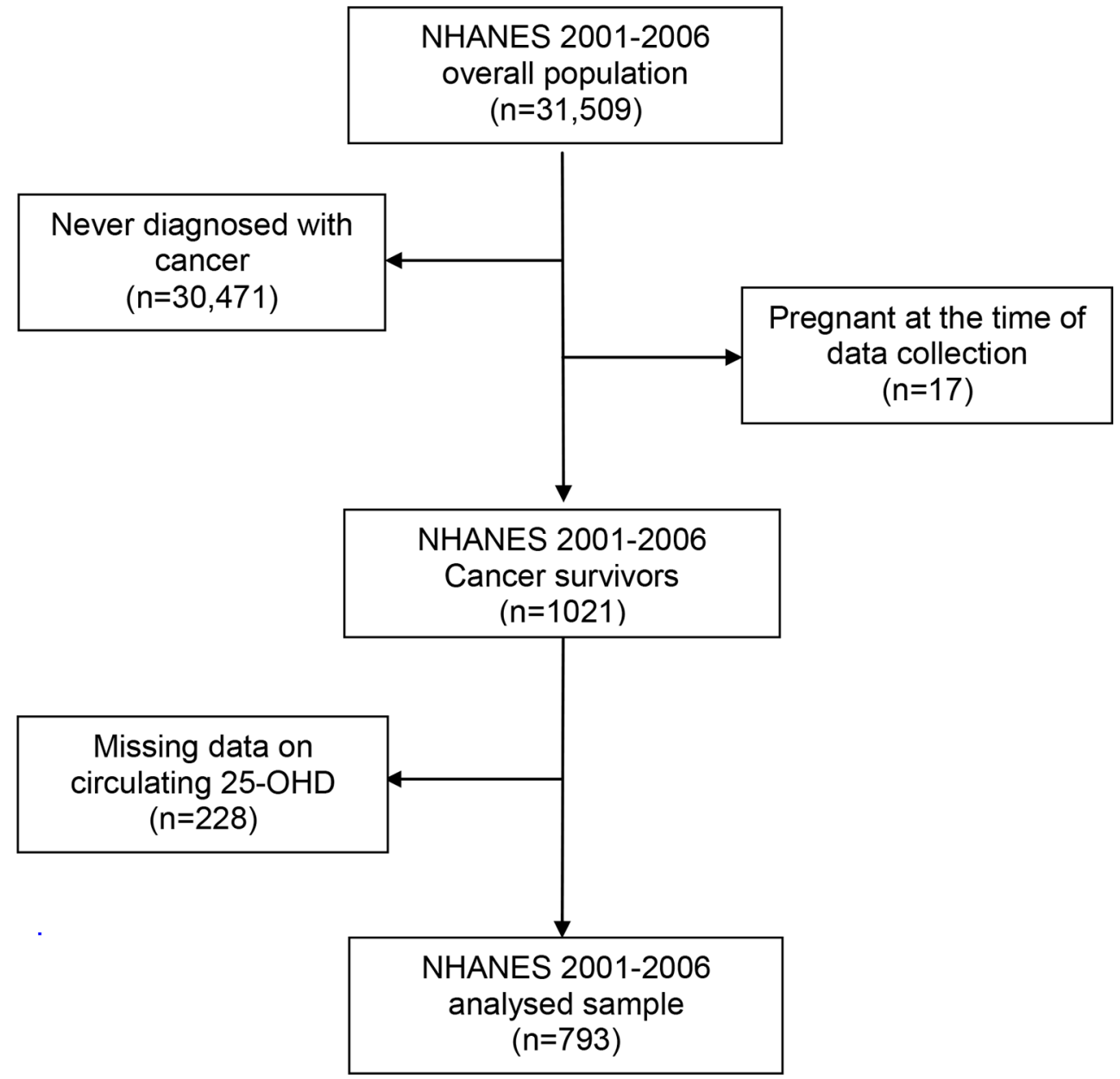

Figure 1 Participants' flow chart: cancer survivors aged 20 years or older from the National Health and Nutrition Examination Survey (NHANES) (2001-2006). 25-OHD, 25-hydroxyvitamin D.

Season of blood draw was determined from the documented month of physical examination. Months were reported in two groups: 1 November through 30 April, or 1 May through 31 October, and classified into winter or summer, respectively. ${ }^{16}$

\section{Dietary vitamin D supplement use}

Information on dietary vitamin D supplement was retrieved from the 30-day dietary supplement data set in the 2001-2006 and 2007-2010 data. In the 2001-2006 data set, we obtained data on individual product for participants who reported taking vitamin supplement, and linked to the Dietary Supplements Ingredient Database. ${ }^{27}$ Products' ingredients that contained vitamin D were aggregated for each participant, and then categorised into a binary variable (yes/no) for dietary vitamin D supplement use assessment. In 2007-2010 data, aggregated information on dietary supplement use (including vitamin D supplement use) was available, thus was used to determine participants' dietary vitamin D supplement use (yes/no).

\section{Self-reported LTPA}

The assessment on self-reported physical activity for 2007-2010 changed from 2005 to 2006 and earlier. There is no conversion provided between the two assessments; therefore, analyses for LTPA were conducted separately for the 2001-2006 and 2007-2010 data.

In the 2001-2006 data, participants self-reported specific LTPA in the past 30 days from a list of 48 activities, if they engaged in certain activities, and the frequencies and durations of these activities. Each activity was coded into a metabolic equivalent task (MET) score based on the 2011 Compendium of Physical Activities, a valid and globally used instrument to quantify the energy expenditure of physical activity in adults. ${ }^{28}$ For each reported activity, MET-minutes per week (MET-min/week) were calculated by multiplying the MET value of each reported activity by the minutes spent in the activity per 7 days. Overall LTPA was summarised as the total MET-min/week of all reported activities. ${ }^{29}$ Cancer survivors were classified as inactive $(0$ MET-min/week), insufficiently active (<750 MET-min/ week) and sufficiently active ( $\geq 750$ MET-min/week) based on the standard definition. ${ }^{29}$ In addition, we categorised each of the 48 listed activities into outdoor (eg, walking, jogging, fishing) or indoor (eg, aerobics, bowling, weights) activity. Activities that could be either indoor or outdoor (eg, bicycling, swimming) were classified as indoor to ensure a conservative estimation of 


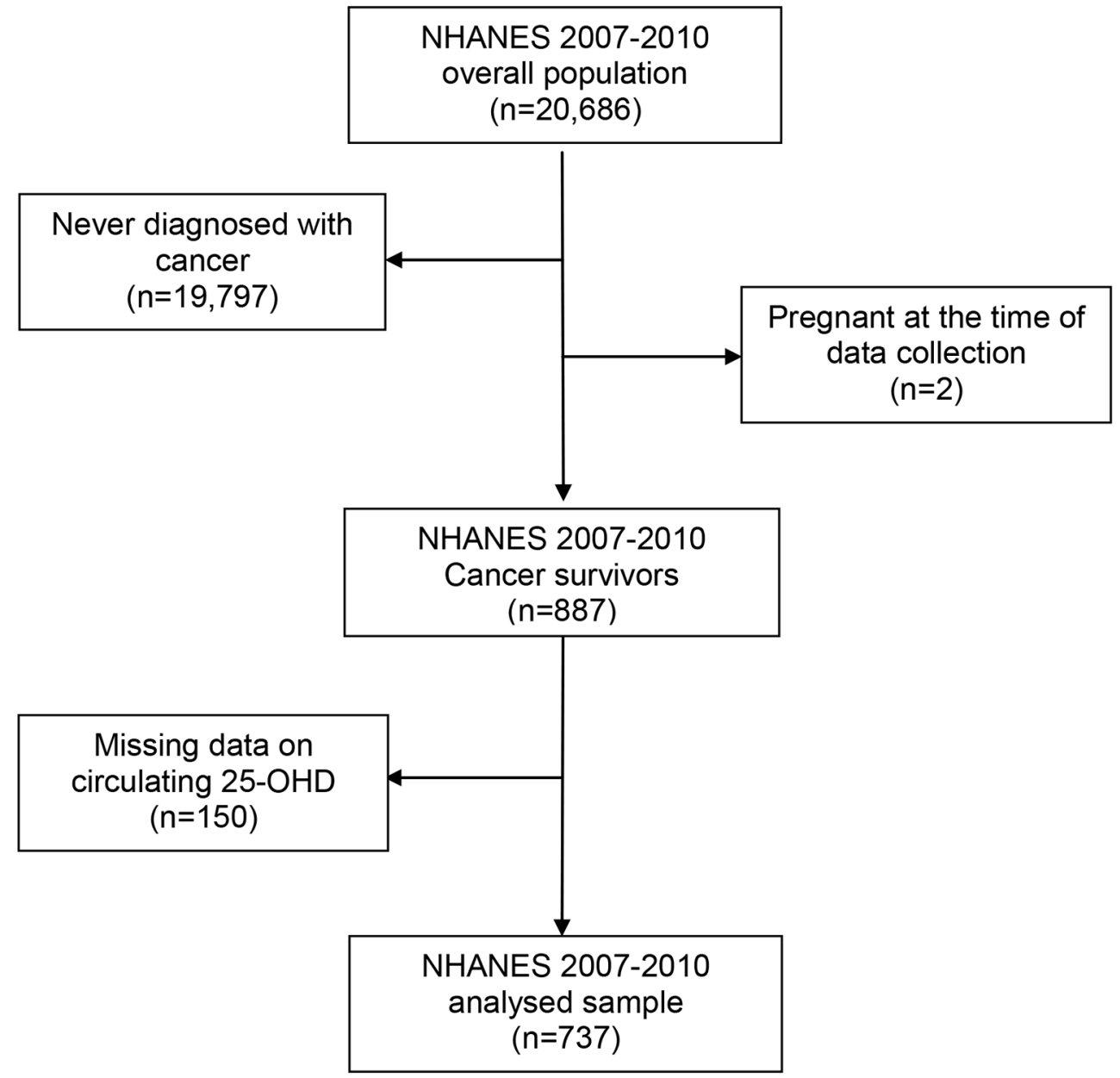

Figure 2 Participants' flow chart: cancer survivors aged 20 years or older from the National Health and Nutrition Examination Survey (NHANES) (2007-2010). 25-OHD, 25-hydroxyvitamin D.

the associations between outdoor LTPA and 25-OHD. Both indoor and outdoor LTPAs were summarised in MET-min/week, then classified as inactive (0 MET-min/ week), insufficiently active ( $<450 \mathrm{MET}-\mathrm{min} /$ week) and sufficiently active ( $\geq 450$ MET-min/week). A cut-off lower than 750 MET-min/week was used for indoor and outdoor activities, given they are subsets of overall LTPA. We used 450 MET-min/week as the cut-off given that it is the minimal goal of weekly LTPA. ${ }^{29}$

In the 2007-2010 data, participants self-reported their daily activities, leisure-time activities and sedentary activities using questions based on the Global Physical Activity Questionnaire. ${ }^{30}$ Levels of LTPA were calculated as the minutes per week that participants reported participating in moderate-to-vigorous-intensity physical activity (MVPA). Participants reported the number of days and minutes spent in moderate recreational and vigorous recreational activities in a typical week by answering the following questions: 'In a typical week, on how many days do you do vigorous-intensity sports, fitness or recreational activities?' 'Minutes vigorous recreational activities', and 'In a typical week, on how many days do you do moderate-intensity sports, fitness or recreational activities?' 'Minutes moderate recreational activities'.
We summarised the total number of minutes for both activities, where the number of minutes spent in vigorous-intensity physical activity was doubled and added to the number of minutes of moderate-intensity physical activity to approximately equal the MET value. ${ }^{31}$ Cancer survivors were classified as inactive (0 min/week MVPA), insufficiently active $(<150 \mathrm{~min} /$ week MVPA) and sufficiently active ( $\geq 150 \mathrm{~min} /$ week MVPA) based on the physical activity guidelines for cancer survivors. ${ }^{32}$

\section{Statistical analysis}

Survey analysis procedures were used to account for the sample weights (MEC exam weight), stratification and clustering of the complex sampling design to ensure nationally representative estimates. Information on sociodemographic characteristics, weight, height, season of blood draw and self-reported LTPA was complete among cancer survivors who had available data on circulating 25-OHD levels. We calculated the descriptive statistics for participants' characteristics and LTPA categories by 25-OHD levels in quintiles separately in 2001-2006 data and 2007-2010 data. We summarised weighted means and SEs for continuous variables, and weighted proportions for categorical variables. 
We estimated linear associations between LTPA and 25-OHD levels in both 2001-2006 and 2007-2010 data. The multivariable linear regression models for LTPA were adjusted for age, sex, race, BMI, smoking status and season of blood draw. In the 2001-2006 data, we further estimated the linear associations between LTPA and 25-OHD separately by indoor and outdoor activities. $X^{2}$ test indicated significant difference $(p<0.001)$ between indoor and outdoor activities. In the multivariable linear regression models, we simultaneously adjusted for both activities. We tested for differences between the indoor and outdoor effects by including both in the regression model and testing for interaction. We examined the normality of residuals by kernel density estimate and standardised normal probability plots for all the linear regression models. Continuous 25-OHD data were categorised as low $(<50 \mathrm{nmol} / \mathrm{L})$ and high $(\geq 50 \mathrm{nmol} / \mathrm{L}) 25$-OHD based on definitions of vitamin $\mathrm{D}$ insufficiency. ${ }^{30}$

To calculate the prevalence ratios (PRs) of high 25-OHD level $(\geq 50 \mathrm{nmol} / \mathrm{L})$ across LTPA categories, we first calculated prevalence ORs (PORs) for each category in multivariable logistic regression models. Since the PORs do not approximate the PRs for common outcome (25-OHD $\geq 50 \mathrm{nmol} / \mathrm{L})$, we used the baseline prevalence to correct the PORs and 95\% CIs based on existing method to obtain reliable PR estimates. ${ }^{33}$ We further conducted the following sensitivity analyses: (1) using BMI as a continuous variable in the regression models; (2) stratification by BMI category; (3) classifying activities that could be either indoor or outdoor (eg, bicycling, swimming) as outdoor activities; and (4) classifying activities that could be either indoor or outdoor (eg, bicycling, swimming) as half-half (MET-min/week) to indoor and outdoor activities.

All statistical significance was set at $\mathrm{p}<0.05$. All statistical analyses were performed using Stata V.14.0.

\section{RESULTS}

Our study population consisted of 1530 cancer survivors who had data on circulating 25-OHD levels. The most prevalent cancer sites were breast cancer (19.3\%), prostate $(18.8 \%)$, cervix $(10.4 \%)$ and colon $(8.6 \%)$. Participants' mean age at the time of baseline examination was 60.5 years, and their mean BMI was $28.6 \mathrm{~kg} /$ $\mathrm{m}^{2}$. Circulating 25-OHD levels were significantly higher among those who reported dietary vitamin D supplement use than those who did not in both 2001-2006 (68.82 vs $56.74 \mathrm{nmol} / \mathrm{L}, \mathrm{p}<0.001)$ and $2007-2010$ data (83.73 vs $60.88 \mathrm{nmol} / \mathrm{L}, \mathrm{p}<0.001)$. We observed statistically significant differences in circulating 25-OHD levels for most characteristics, except for age and sex (2001-2006 in table 1 and 2007-2010 in table 2). Cancer survivors who were obese, non-Hispanic black or smokers had lower 25-OHD levels than those who had normal weight, were non-Hispanic white/Hispanic and were non-smokers, respectively.

\section{Associations between LTPA and circulating 25-OHD levels}

Tables 3 and 4 summarise both the non-adjusted and adjusted associations between LTPA and circulating 25-OHD in linear regression and logistic regression models, respectively. Because LTPA measure differed between 2001-2006 and 2007-2010 and there is no conversion between the two, it is not possible to compare the findings between two study phases directly. Cancer survivors who were sufficiently active had higher circulating 25-OHD levels than those who were inactive in univariate analyses, and these findings were maintained in multivariable analyses in the 2001-2006 data, but not the 2007-2010 data. This translated to $8.07 \mathrm{nmol} / \mathrm{L}(95 \% \mathrm{CI}$ 4.63 to 11.52) higher 25-OHD levels in 2001-2006 phase in the multivariable-adjusted models. Furthermore, the comprehensive data on a list of 48 activities collected in 2001-2006 allowed us to extend the analyses to compare between indoor and outdoor LTPA in relation to 25-OHD levels. In the non-adjusted models (table 3), higher levels of indoor and outdoor LTPA both were associated with higher 25-OHD levels. However, in multivariable-adjusted models (that also mutually adjusted for indoor and outdoor LTPA), the association was only statistically significant among cancer survivors who engaged in outdoor LTPA (5.83 nmol/L, 95\% CI 1.64 to 10.01). The interaction between indoor and outdoor activities was not significant $(\mathrm{p}=0.29)$. Analyses using logistic regression models were supportive. Our findings were similar when we classified activities that could be either indoor or outdoor (eg, bicycling, swimming) as outdoor activities $(6.39 \mathrm{nmol} / \mathrm{L}, 95 \%$ CI 2.85 to 9.94$)$, and classifying these activities as half-half (MET-min/week) to indoor and outdoor activities $(7.26 \mathrm{nmol} / \mathrm{L}, 95 \%$ CI 2.88 to 11.64$)$ (data not shown).

Likewise, we observed similar results in sensitivity analyses using BMI as a continuous variable; higher 25-OHD levels were associated with LTPA in the overall analyses $(7.74 \mathrm{nmol} / \mathrm{L}, 95 \%$ CI 4.53 to 10.95$)$ and among those who engaged in outdoor LTPA $(5.82 \mathrm{nmol} / \mathrm{L}, 95 \% \mathrm{CI}$ 1.69 to 9.95 ) (data not shown). In stratified analyses, associations of LTPA with higher circulating 25-OHD levels were retained in the obese group in the 2001-2006 data (7.10 nmol/L, 95\% CI 2.51 to 11.70 , outdoor LTPA) as well as $2007-2010$ data $(13.91 \mathrm{nmol} / \mathrm{L}, 95 \%$ CI 3.86 to 23.96 , overall LTPA) (see online supplementary 1). The stratified analyses should, however, be interpreted cautiously because the relatively small number of participants in the different strata may not allow for very robust effect estimates. Outdoor LTPA was lower in non-Hispanic black (69.2\% inactive vs $51.5 \%$ inactive among non-Hispanic whites, and $43.2 \%$ inactive among Hispanics) (data not shown).

\section{DISCUSSION}

We observed that being physically active was associated with higher circulating 25-OHD levels in a nationally representative sample of cancer survivors. Further 


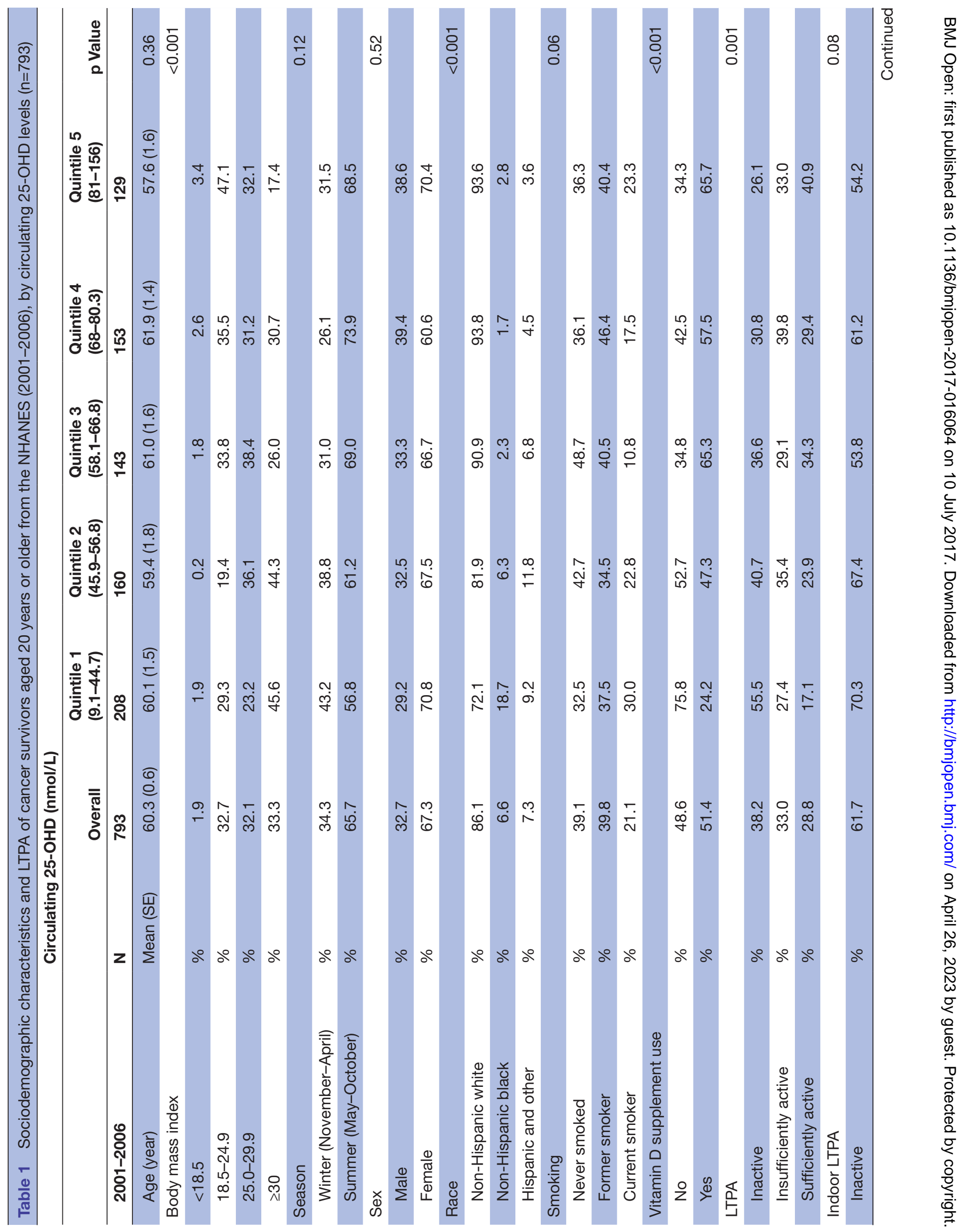




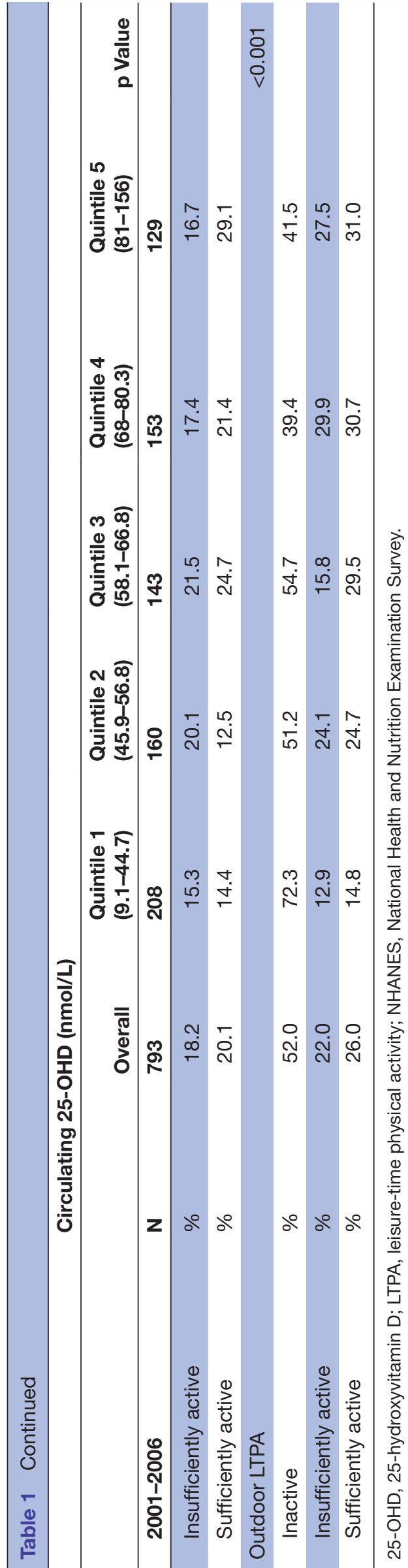

analyses showed that the elevated 25-OHD levels were only statistically significant among cancer survivors who engaged in outdoor physical activity.

To the best of our knowledge, this is the first study to evaluate the associations of physical activity with circulating 25-OHD levels in cancer survivors. Our findings are, however, similar to what has been reported among non-cancer participants enrolled in NHANES (19881994). ${ }^{16}$ Scragg and Camargo reported a $9.6 \mathrm{nmol} / \mathrm{L}$ increase in 25-OHD levels among participants who engaged in outdoor LTPA compared with those who did not engage in outdoor LTPA. The increase in 25-OHD levels associated with outdoor LTPA is higher than what we observed in our study population $(5.83 \mathrm{nmol} / \mathrm{L}$ higher 25-OHD). This could be due to the different ways LTPA was categorised. The most active group in their study translates to participating daily in outdoor activity, while only $5.6 \%$ (weighted proportion) of cancer survivors in our sample achieved this physical activity level. To compare at an equivalently active level, our findings of a $5.83 \mathrm{nmol} / \mathrm{L}$ increase in cancer survivors are similar to $6.1 \mathrm{nmol} / \mathrm{L}$ higher 25-OHD level in individuals who were at a similar activity level (engaged in 13-30 times outdoor LTPA per month) reported by Scragg and Camargo. ${ }^{16}$ Data from trials have shown that each 40 IU of vitamin D consumed increases serum 25-OHD concentrations by $0.53 \mathrm{nmol} / \mathrm{L}$ in adults. ${ }^{34}$ The recommended dietary vitamin $\mathrm{D}$ allowance for adults in the USA is $600 \mathrm{IU}$, which is expected to increase circulating 25-OHD levels by $15 \mathrm{nmol} / \mathrm{L}$. Thus, our findings (a $5.83 \mathrm{nmol} / \mathrm{L}$ increase) suggest that engaging in outdoor LTPA could provide clinically meaningful increases in 25-OHD levels among cancer survivors. A more recent analysis using NHANES 2003-2006 data reported increasing level of 25-OHD is associated with higher level of objectively measured moderate-to-vigorous physical activity, but the association was not stronger for outdoor LTPA compared with indoor when using self-reported data. ${ }^{17}$

It is unclear whether physical activity has direct or indirect effects on 25-OHD levels. Sun exposure is the major determinant of circulating 25-OHD levels; hence, it is possible that physical activity may indirectly impact 25-OHD levels through increased sun exposure associated with outdoor activity ${ }^{7}$ among active individuals, yet few studies have measured activities specifically to outdoor, or able to adjusted for sun exposure. ${ }^{16} 173536$ On the other hand, physical activity may directly impact 25-OHD metabolism. Zittermann and colleagues ${ }^{18}$ reported higher calcium absorption rates and plasma calcritrol levels in exercise-trained young men compared with age-matched sedentary controls. Similarly, in a small study, young men who underwent muscle-building exercise (indoor) for at least 1 year had higher circulating 25-OHD, Gla protein and 1,25-dihydroxyvitamin levels compared with age-matched controls who received constant daily diet same as the exercise group. ${ }^{20}$ However, whether this mechanism operates in cancer survivors is unclear because of the physiological, biological and 


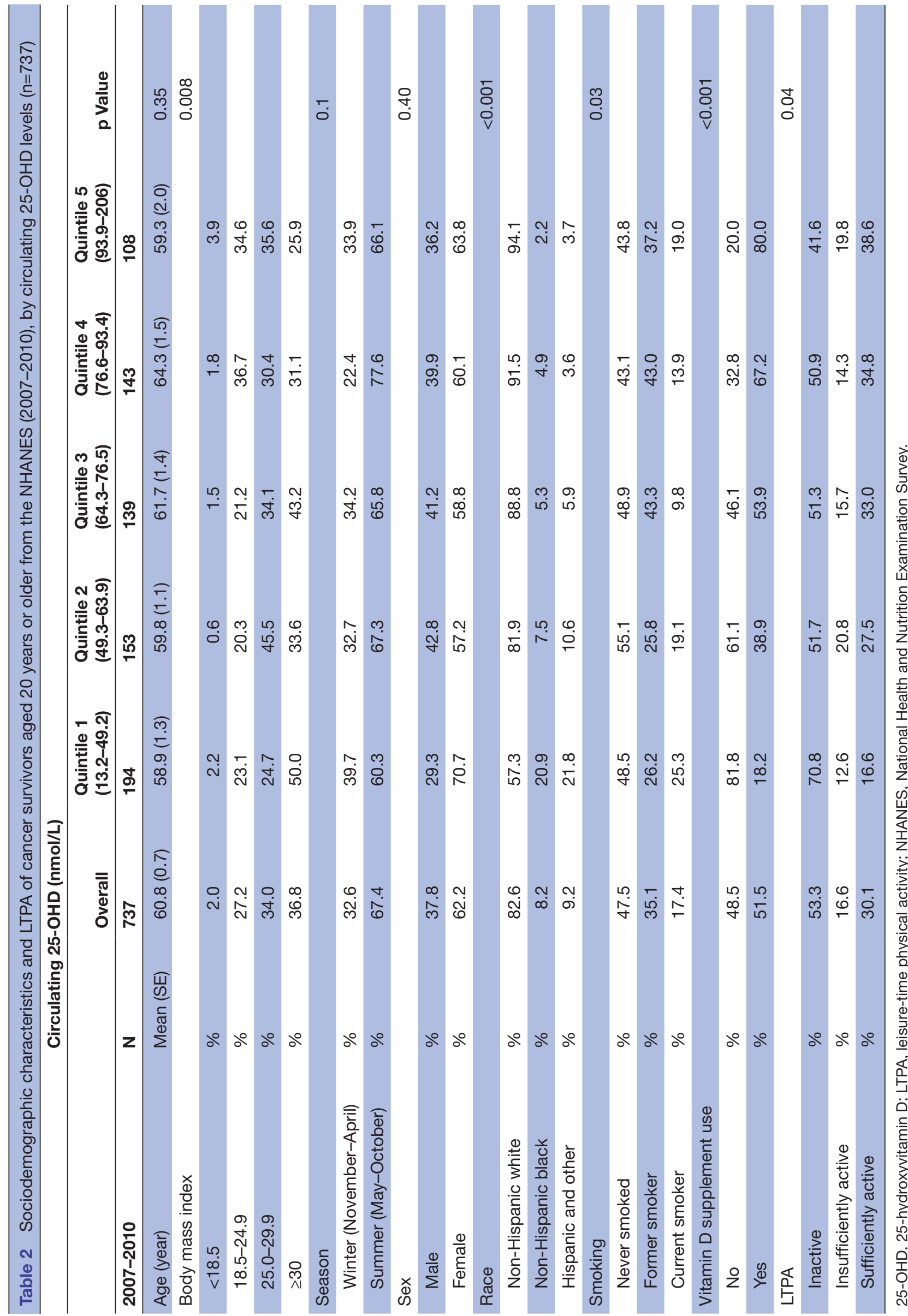


Table 3 Associations between LTPA and circulating 25-OHD level from unadjusted and multivariable linear regression models among cancer survivors aged 20 years or older from the NHANES (2001-2010)

\section{Circulating 25-OHD (nmol/L)}

2001-2006* $(n=793)$

UnadjustedBeta coefficient $(95 \% \mathrm{Cl})$

Adjusted†Beta coefficient $(95 \% \mathrm{Cl})$

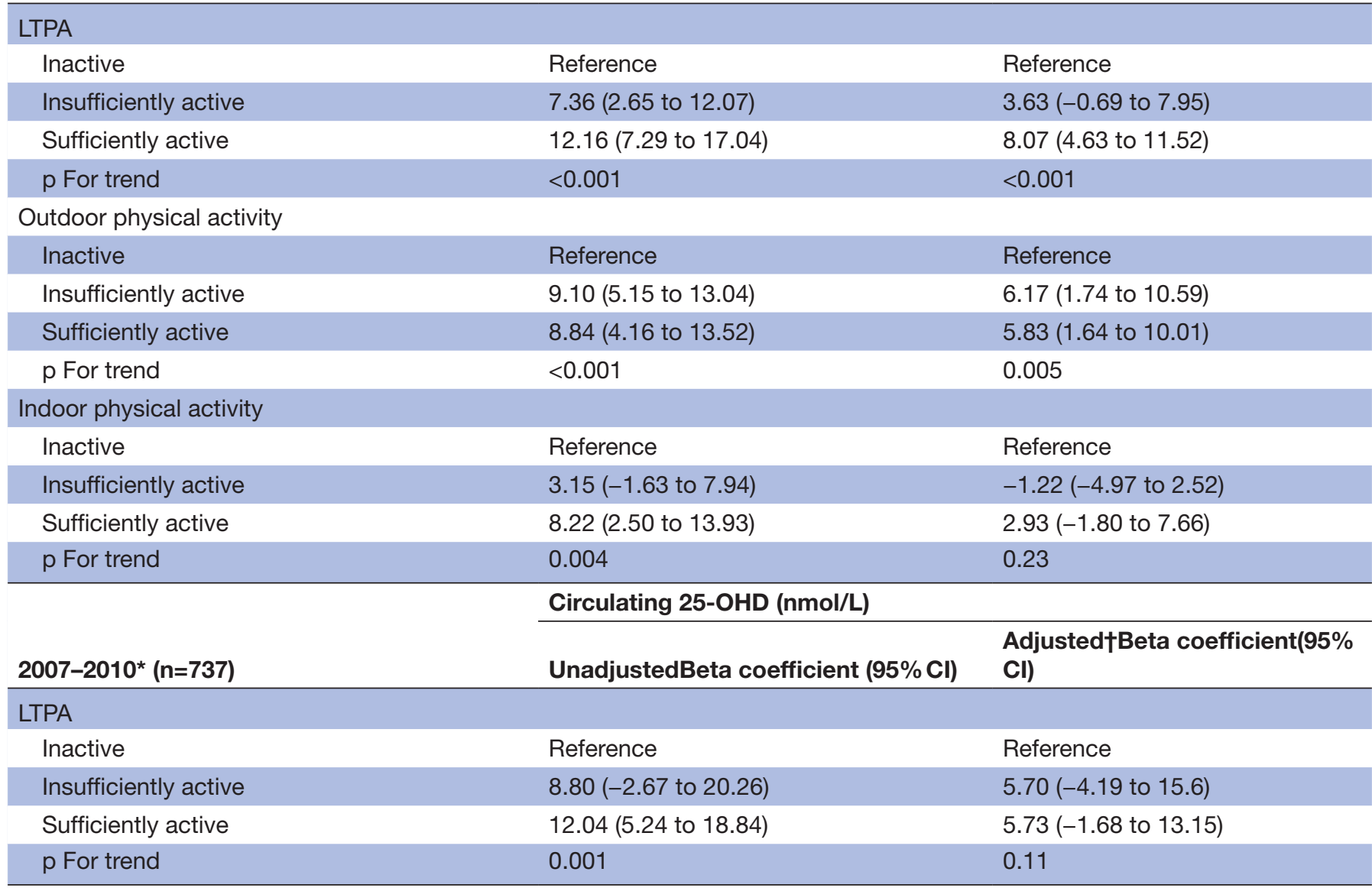

*LTPA data analysed separately due to the changes in self-reported LTPA measures from wave 2005-2006 to wave 2007-2008.

†Adjusted for age, sex, race, body mass index, smoking status and dietary vitamin D supplement use.

25-OHD, 25-hydroxyvitamin D; LTPA, leisure-time physical activity; NHANES, National Health and Nutrition Examination Survey.

behavioural alterations associated with cancer and cancer treatment. $^{32}$

We observed statistically significant higher circulating 25-OHD levels associated with outdoor, but not with indoor, LTPA in the mutually adjusted model. Nevertheless, no statistically significant interaction between indoor and outdoor LTPA was observed. It is likely that LTPA influences 25-OHD via multiple pathways, possibly both an indirect effect due to sun exposure and a direct impact on 25-OHD metabolism. However this warrants further investigation using precise measures of physical activity ${ }^{37}$ and taking into consideration sun exposure and other vitamin $\mathrm{D}$ metabolites.

We observed that obese cancer survivors who were active had higher circulating 25-OHD levels. Obesity is believed to induce low circulating 25-OHD levels through volumetric dilution of vitamin $\mathrm{D}$ in the excessive adipose tissue. ${ }^{38}$ Given that obese cancer survivors are at higher risk of vitamin D deficiency compared with the non-obese, ${ }^{39} 40$ the present findings suggested engaging in physical activity might be particularly important to maintain or increase circulating 25-OHD levels among obese cancer survivors. Future studies are needed to confirm these findings using more precise measures of adiposity (eg, body fat percentage) in a larger study population.

The association between LTPA and dietary vitamin D supplement use appeared to differ between 2001-2006 data $(p=0.19)$ and 2007-2010 data $(p=0.03)$, although the prevalence of dietary vitamin $\mathrm{D}$ supplement use was similar in two study phases $(51.4 \%$ vs $51.5 \%)$. In the 2007-2010 data, active cancer survivors are more likely to report dietary vitamin D supplement use compared with inactive ones. Thus, the non-significant findings of LTPA and circulating 25-OHD levels could arise from the change in self-reported LTPA measures from 2001-2006 to 2007-2010 data.

The main strength of this analysis is pooling cancer survivors from a nationally representative adult sample in the USA. We aggregated five waves' data and achieved a fairly sizeable sample. In addition, we controlled for a 
Table 4 Associations between LTPA and circulating 25-OHD level from unadjusted and adjusted logistic regression models among cancer survivors aged 20 years or older from the NHANES (2001-2010)

\begin{tabular}{|c|c|c|}
\hline 2001-2006* & \multicolumn{2}{|c|}{ Circulating $25-\mathrm{OHD} \geq 50 \mathrm{nmol} / \mathrm{L}(\mathrm{n}=534)$} \\
\hline $\begin{array}{l}\text { Reference Circulating } 25-\mathrm{OHD}<50 \mathrm{nmol} / \mathrm{L} \\
(\mathrm{n}=259)\end{array}$ & $\begin{array}{l}\text { UnadjustedPrevalence ratio } \\
(95 \% \mathrm{Cl}) \dagger\end{array}$ & $\begin{array}{l}\text { Adjusted } \neq \text { Prevalence ratio } \\
(95 \% \mathrm{Cl}) \dagger\end{array}$ \\
\hline \multicolumn{3}{|l|}{ LTPA } \\
\hline Inactive & Reference & Reference \\
\hline Insufficiently active & 1.19 (1.02 to 1.33$)$ & 1.10 (0.88 to 1.27$)$ \\
\hline Sufficiently active & 1.36 (1.30 to 1.45$)$ & $1.32(1.19$ to 1.41$)$ \\
\hline $\mathrm{p}$ For trend & $<0.001$ & $<0.001$ \\
\hline \multicolumn{3}{|l|}{ Outdoor physical activity } \\
\hline Inactive & Reference & Reference \\
\hline Insufficiently active & $1.21(1.10$ to 1.30$)$ & 1.16 (1.01 to 1.27$)$ \\
\hline Sufficiently active & 1.24 (1.11 to 1.33$)$ & 1.22 (1.06 to 1.32$)$ \\
\hline $\mathrm{p}$ For trend & 0.001 & 0.009 \\
\hline \multicolumn{3}{|l|}{ Indoor physical activity } \\
\hline Inactive & Reference & Reference \\
\hline Insufficiently active & 1.19 (0.99 to 1.33$)$ & 1.10 (0.87 to 1.27$)$ \\
\hline Sufficiently active & 1.21 (1.05 to 1.33$)$ & 1.07 (0.88 to 1.23$)$ \\
\hline $\mathrm{p}$ For trend & 0.006 & 0.32 \\
\hline 2007-2010* & \multicolumn{2}{|c|}{ Circulating $25-\mathrm{OHD} \geq 50 \mathrm{nmol} / \mathrm{L}(\mathrm{n}=531)$} \\
\hline $\begin{array}{l}\text { Reference Circulating 25-OHD }<50 \mathrm{nmol} / \mathrm{L} \\
(\mathrm{n}=206)\end{array}$ & $\begin{array}{l}\text { UnadjustedPrevalence ratio } \\
(95 \% \mathrm{Cl})\end{array}$ & $\begin{array}{l}\text { Adjusted†Prevalence ratio } \\
(95 \% \mathrm{Cl})\end{array}$ \\
\hline \multicolumn{3}{|l|}{ LTPA } \\
\hline Inactive & Reference & Reference \\
\hline Insufficiently active & 1.15 (0.97 to 1.26$)$ & $1.14(0.92$ to 1.27$)$ \\
\hline Sufficiently active & 1.22 (1.07 to 1.30$)$ & 1.13 (0.90 to 1.27$)$ \\
\hline $\mathrm{p}$ For trend & 0.008 & 0.18 \\
\hline
\end{tabular}

*LTPA data analysed separately due to the changes in self-reported LTPA measures from wave 2005-2006 to wave 2007-2008.

†Prevalence ratio and $95 \%$ Cls were corrected using prevalence OR and prevalence of high $25-\mathrm{OHD}$ level $(\geq 50 \mathrm{nmol} / \mathrm{L})$ in reference groups. $\ddagger$ Adjusted for age, sex, race, body mass index, smoking status and dietary vitamin $D$ supplement use.

25-OHD, 25-hydroxyvitamin D; LTPA, leisure-time physical activity; NHANES, National Health and Nutrition Examination Survey.

range of factors that are known to affect the circulating 25-OHD levels. Further, we were able to compare associations of LTPA with 25-OHD by outdoor and indoor LTPA, thereby providing further insights on the associations of LTPA with 25-OHD levels.

There are a number of limitations to this study. First, the cross-sectional nature of this study makes it impossible to determine a causal association. The debate on whether vitamin D deficiency is a risk factor for mortality or an indicator of good health is ongoing. ${ }^{412}$ It is possible that active cancer survivors were more active because of better health status than those who were inactive. Thus, the higher 25-OHD levels in active cancer survivors might be an indicator of better overall health. Second, season, an important determinant of 25-OHD levels, was only available in two categories. Solar radiation, required for skin to synthesise vitamin $\mathrm{D}$, is weaker in winter compared with summer. However, there were no statistically significant differences between winter (southern states) and summer (northern states) 25-OHD levels in our study population, probably owing to the timing of blood collection in each region. The NHANES study collected blood samples in the southern states during winter and in the northern states during summer. Third, we were not able to conduct analyses stratified by cancer type or time since diagnosis because of the limited number of individual cancers. Finally, physical activity was self-reported. Participants who received chemotherapy within the last 4 weeks were excluded from blood collection within the NHANES study. Chemotherapy-associated reduction of circulating 25-OHD level has been documented previously. ${ }^{43-45}$ Therefore our findings might not be generalisable to patients receiving chemotherapy.

Our findings of an association between LTPA and 25-OHD, which was stronger for outdoor LTPA compared with indoor LTPA, have implications for public health recommendations in cancer survivors. Although the casual relationship of 25-OHD with cancer survival is yet 
unclear, strong evidence supports the benefits of physical activity in improved cancer survival and the quality of life during survival. ${ }^{37} 46$ Our findings suggest that 25-OHD might be a surrogate marker of physical activity that accounts for the direct and indirect effects of LTPA, particularly outdoor. ${ }^{716}$ The proportion of cancer survivors in NHANES who did not engage in any LTPA was high, especially in the 2007-2010 (53.3\%) compared with the 2001-2006 wave $(38.3 \%)$. This observed decline in LTPA might be attributed to the differences in measures and may not reflect an actual change in LTPA levels, that is, the 2001-2006 measure comprised 48 activity items while the 2007-2010 measure queries general physical activity participation. These differences in measures may also contribute to the non-significant findings observed in the 2007-2010 data. In fact, an increase in the physical activity level in the US population from 2001 to 2011 has been reported from the The Behavioral Risk Factor Surveillance System (BRFSS) data ${ }^{47}$ although this trend may not hold true in cancer survivors. Guidelines from the American Cancer Society ${ }^{32}$ and American College of Sports Medicine ${ }^{48}$ suggest that cancer survivors should follow the physical activity guidelines for Americans with specific exercise programming adaptations based on disease-related and treatment-related adverse effects. However, physical activity levels in these populations are critically low during and after treatment. ${ }^{49}$ Physical activity interventions in cancer survivors may consider including early morning (before 11:00) outdoor activities for about $15 \mathrm{~min}$. Notably, given the well-documented differences in cancer prognosis between non-Hispanic blacks and other racial/ethnic groups, and the emerging associations of vitamin $\mathrm{D}$ with cancer prognosis, physical activity interventions incorporating outdoor activities might be particularly important for cancer survival among non-Hispanic blacks.

In conclusion, physical activity, particularly outdoor physical activity, is associated with higher 25-OHD levels in cancer survivors. This adds to the potential health benefits of being physically active. Non-Hispanic black cancer survivors, who are more likely to have vitamin D deficiency, were less likely to engage in outdoor LTPA. In view of the possible beneficial effects of vitamin $\mathrm{D}$ on cancer prognosis, engaging in outdoor physical activity could provide clinically meaningful increases in 25-OHD levels among cancer survivors.

Contributors LY and ATT conceived and designed the study, analysed and interpreted data, and drafted and reviewed the manuscript.

Funding This work was supported by the Transdisciplinary Research on Energetics and Cancer (TREC) Center at Washington University in St Louis (LY), and the Alvin J Siteman Cancer Center, Barnes-Jewish Hospital Foundation and Washington University School of Medicine (ATT). The TREC Center is funded by the National Cancer Institute at NIH (U54 CA155496).

Competing interests None declared.

Ethics approval National Center for Health Statistics Research Ethics Review Board Approval.

Provenance and peer review Not commissioned; externally peer reviewed.
Data sharing statement The NHANES data are publicly available at ttps://wwwn. cdc.gov/nchs/nhanes/Default.aspx.

Open Access This is an Open Access article distributed in accordance with the Creative Commons Attribution Non Commercial (CC BY-NC 4.0) license, which permits others to distribute, remix, adapt, build upon this work non-commercially, and license their derivative works on different terms, provided the original work is properly cited and the use is non-commercial. See: http://creativecommons.org/ licenses/by-nc/4.0/

(C) Article author(s) (or their employer(s) unless otherwise stated in the text of the article) 2017. All rights reserved. No commercial use is permitted unless otherwise expressly granted.

\section{REFERENCES}

1. Miller KD, Siegel RL, Lin CC, et al. Cancer treatment and survivorship statistics, 2016. CA Cancer J Clin 2016;66:271-89.

2. Toriola AT, Nguyen N, Scheitler-Ring K, et al. Circulating 25-hydroxyvitamin $\mathrm{D}$ levels and prognosis among Cancer patients: a systematic review. Cancer Epidemiol Biomarkers Prev 2014;23:917-33

3. Yao S, Kwan ML, Ergas IJ, et al. Association of serum level of vitamin $\mathrm{D}$ at diagnosis with breast Cancer survival: a Case-Cohort analysis in the Pathways Study. JAMA oncology 2016.

4. Mondul AM, Weinstein SJ, Moy KA, et al. Circulating 25-Hydroxyvitamin D and Prostate Cancer Survival. Cancer Epidemiol Biomarkers Prev 2016;25:665-9.

5. Webb PM, de Fazio A, Protani MM, et al. Circulating 25-hydroxyvitamin D and survival in women with ovarian Cancer. Am J Clin Nutr 2015;102:109-14.

6. Heaney RP. Serum 25-hydroxyvitamin D is a reliable indicator of vitamin D status. Am J Clin Nutr 2011;94:619-20.

7. Holick MF. Vitamin D deficiency. N Engl J Med 2007;357:266-81.

8. Holick MF, Binkley NC, Bischoff-Ferrari HA, et al. Evaluation, treatment, and prevention of vitamin $\mathrm{D}$ deficiency: an Endocrine Society clinical practice guideline. J Clin Endocrinol Metab 2011;96:1911-30.

9. Sowers MR, Wallace RB, Hollis BW, et al. Parameters related to 25$\mathrm{OH}-\mathrm{D}$ levels in a population-based study of women. Am J Clin Nutr 1986;43:621-8.

10. Ulrich CM, Holmes RS. Shedding light on colorectal cancer prognosis: vitamin d and beyond. J Clin Oncol 2008;26:2937-9.

11. Autier P, Boniol M, Pizot C, et al. Vitamin D status and ill health: a systematic review. Lancet Diabetes Endocrinol 2014;2:76-89.

12. Ballard-Barbash R, Friedenreich CM, Courneya KS, et al. Physical activity, biomarkers, and disease outcomes in cancer survivors: a systematic review. J Natl Cancer Inst 2012;104:815-40.

13. Schmid D, Leitzmann MF. Association between physical activity and mortality among breast Cancer and colorectal Cancer survivors: a systematic review and meta-analysis. Ann Oncol 2014;25:1293-311.

14. Arem H, Moore SC, Park Y, et al. Physical activity and cancerspecific mortality in the NIH-AARP Diet and Health Study cohort. Int $J$ Cancer 2014;135:423-31.

15. Brock K, Huang WY, Fraser DR, et al. Low vitamin D status is associated with physical inactivity, obesity and low vitamin D intake in a large US sample of healthy middle-aged men and women. $J$ Steroid Biochem Mol Biol 2010;121:462-6.

16. Scragg $R$, Camargo $C A$. Frequency of leisure-time physical activity and serum 25-hydroxyvitamin D levels in the US population: results from the Third National Health and Nutrition Examination Survey. Am J Epidemiol 2008;168:577-86.

17. Wanner M, Richard A, Martin B, et al. Associations between objective and self-reported physical activity and vitamin $D$ serum levels in the US population. Cancer Causes Control 2015;26:881-91.

18. Zittermann A, Sabatschus O, Jantzen S, et al. Exercise-trained young men have higher calcium absorption rates and plasma calcitriol levels compared with age-matched sedentary controls. Calcif Tissue Int 2000;67:215-9.

19. Yeh JK, Aloia JF, Yasumura S, et al. Effect of physical activity on calcium and phosphorus metabolism in the rat. Am J Physiol 1989;256(1 Pt 1):E1-6.

20. Bell NH, Godsen RN, Henry DP, et al. The effects of muscle-building exercise on vitamin D and mineral metabolism. J Bone Miner Res 1988;3:369-74.

21. Klausen T, Breum L, Sørensen HA, et al. Plasma levels of parathyroid hormone, vitamin $\mathrm{D}$, calcitonin, and calcium in association with endurance exercise. Calcif Tissue Int 1993;52:205-8. 
22. Teleni L, Baker J, Koczwara B, et al. Clinical outcomes of vitamin $\mathrm{D}$ deficiency and supplementation in Cancer patients. Nutr Rev 2013;71:611-21.

23. Churilla TM, Lesko SL, Brereton HD, et al. Serum vitamin D levels among patients in a clinical oncology practice compared to primary care patients in the same community: a case-control study. BMJ Open 2011;1:e000397.

24. Centers for Disesae Control and Prevention. National Health and Nutrition Examination Survey. http://www.cdc.gov/nchs/nhanes.htm (accessed 21 Jun 2016)

25. Centers for Disesae Control and Prevention. NHANES Laboratory/ Medical Technologists Procedures Manua. 2009. https://www.cdc. gov/nchs/data/nhanes/nhanes_09_10/lab.pdf (accessed 21 Jun 2016).

26. Centers for Disesae Control and Prevention. Analytical note for 25-Hydroxyvitamin D Data analysis using NHANES III (1988-1994), NHANES 2001-2006, and NHANES 2007-2010, 2015. http://wwwn cdc.gov/nchs/nhanes/VitaminD/AnalyticalNote.aspx. (accessed 21 Jun 2016).

27. Dwyer JT, Picciano MF, Betz JM, et al. Progress in development of an integrated dietary supplement ingredient database at the NIH Office of Dietary Supplements. J Food Compost Anal 2006;19:S108-S114.

28. Ainsworth BE, Haskell WL, Herrmann SD, et al. 2011 compendium of physical activities: a second update of codes and MET values. Med Sci Sports Exerc 20112011;43:1575-81;43:1575-81.

29. Navaneethan SD, Kirwan JP, Arrigain S, et al. Overweight, obesity and intentional weight loss in chronic kidney disease: nhanes 19992006. Int J Obes 2012;36:1585-90.

30. Hallal PC, Andersen LB, Bull FC, et al. Global physical activity levels: surveillance progress, pitfalls, and prospects. Lancet 2012;380:247-57.

31. Zhao G, Li C, Ford ES, et al. Leisure-time aerobic physical activity, muscle-strengthening activity and mortality risks among US adults: the NHANES linked mortality study. Br J Sports Med 2014;48:244-9.

32. Rock CL, Doyle C, Demark-Wahnefried W, et al. Nutrition and physical activity guidelines for Cancer survivors. CA Cancer J Clin 2012;62:242-74.

33. Zhang J, Yu KF. What's the relative risk? A method of correcting the odds ratio in cohort studies of common outcomes. JAMA 1998;280:1690-1.

34. Seamans KM, Cashman KD. Existing and potentially novel functional markers of vitamin D status: a systematic review. Am J Clin Nutr 2009;89:1997S-2008.

35. Scragg R, Holdaway I, Singh V, et al. Serum 25-hydroxyvitamin D3 is related to physical activity and ethnicity but not obesity in a multicultural workforce. Aust N Z J Med 1995;25:218-23.
36. Florez $\mathrm{H}$, Martinez R, Chacra W, et al. Outdoor exercise reduces the risk of hypovitaminosis D in the obese. J Steroid Biochem Mol Biol 2007;103:679-81.

37. Skender S, Schrotz-King P, Böhm J, et al. Repeat physical activity measurement by accelerometry among colorectal Cancer patients-feasibility and minimal number of days of monitoring. BMC Res Notes 2015:8:222

38. Drincic AT, Armas LA, Van Diest EE, et al. Volumetric dilution, rather than sequestration best explains the low vitamin $D$ status of obesity. Obesity 2012;20:1444-8.

39. Friedman CF, DeMichele A, Su HI, et al. Vitamin d deficiency in postmenopausal breast cancer survivors. J Womens Health 2012;21:456-62.

40. Vashi PG, Lammersfeld CA, Braun DP, et al. Serum 25-hydroxyvitamin $D$ is inversely associated with body mass index in Cancer. Nutr J 2011;10:51.

41. Schöttker B, Jorde R, Peasey A, et al. Vitamin D and mortality: meta-analysis of individual participant data from a large consortium of cohort studies from Europe and the United States. BMJ 2014;348:g3656.

42. Chowdhury R, Kunutsor S, Vitezova A, et al. Vitamin D and risk of cause specific death: systematic review and meta-analysis of observational cohort and randomised intervention studies. BMJ 2014;348:g1903.

43. Fakih MG, Trump DL, Johnson CS, et al. Chemotherapy is linked to severe vitamin $\mathrm{D}$ deficiency in patients with colorectal Cancer. Int J Colorectal Dis 2009;24:219-24.

44. Fakih MG, Andrews C, McMahon J, et al. A prospective clinical trial of cholecalciferol 2000 IU/day in colorectal cancer patients: evidence of a chemotherapy-response interaction. Anticancer Res 2012;32:1333-8.

45. Jacot W, Pouderoux S, Thezenas S, et al. Increased prevalence of vitamin $D$ insufficiency in patients with breast cancer after neoadjuvant chemotherapy. Breast Cancer Res Treat 2012;134:709-17.

46. Strasser B, Steindorf K, Wiskemann J, et al. Impact of resistance training in Cancer survivors. Medicine \& Science in Sports \& Exercise 2013;45:2080-90.

47. Dwyer-Lindgren L, Freedman G, Engell RE, et al. Prevalence of physical activity and obesity in US counties, 2001-2011: a road map for action. Popul Health Metr 2013;11:7.

48. Schmitz KH, Courneya KS, Matthews C, et al. American College of Sports Medicine Roundtable on Exercise guidelines for Cancer survivors. Medicine \& Science in Sports \& Exercise 2010;42:1409-26.

49. Huy C, Schmidt ME, Vrieling A, et al. Physical activity in a german breast Cancer patient cohort: one-year trends and characteristics associated with change in activity level. Eur J Cancer 2012;48:297-304. 